\title{
PRESSURE OSCILLATION IN RHIC CRYOGENIC SYSTEM
}

\author{
L. X. Jia, C. Montag, T. Tallerico, W. Hirzel, and A. Nicoletti \\ Brookhaven National Laboratory \\ Upton, New York 11973, USA
}

\begin{abstract}
Horizontal beam vibration around $10 \mathrm{~Hz}$ in the Relativistic Heavy Ion Collider (RHIC) have been identified and the possible sources to cause this vibration have been investigated. To determine the helium pressure oscillations as a possible primary vibration source, helium pressure measurements were carried out in the five cryogenic transfer lines at two valve boxes and six lead ports at one triplet cryostat for both magnet rings. Additionally, cold masses inside one triplet cryostat have also been equipped with accelerometers to further investigate the phenomenon. This paper reports the helium pressure oscillations, including one at $10 \mathrm{~Hz}$ with peak-to-peak pressure of $7 \mathrm{kPa}$, which is close to the frequency of beam vibration.
\end{abstract}

\section{INTRODUCTION}

The Relativistic Heavy Ion Collider (RHIC) at Brookhaven National Laboratory consists of two superconducting magnet storage rings ("Blue" and "Yellow") that intersect at six equidistantly-distributed locations around the $3.8 \mathrm{~km}$ machine circumference, as shown in FIGURE 1. The two RHIC rings share 6 common magnet cryostats ("Triplet") at beam interaction region. Each triplet cryostat contains the cold masses of three quadrupoles (Q1, Q2, and Q3) and one dipole (D0) per ring, eight cold masses in total, which are separate for both beams but share a common cryostat, see

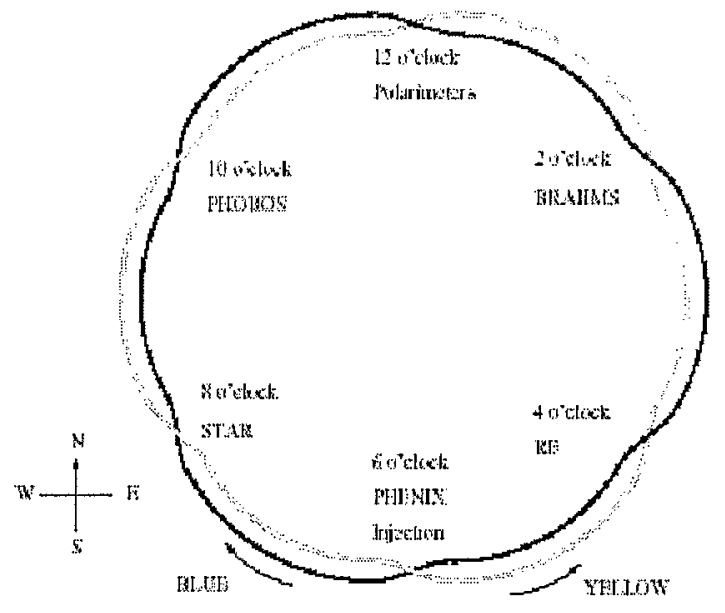

FIGURE 1 Six IP's in two RHIC magnet rings of blue and yellow. 
FIGURE 2. The cold mass of three quadrupoles for each ring is suspended on two posts, see FIGURE 3. The connection between cold masses of two rings is designed to allow independent transverse motion, while it is stiff in the longitudinal direction. Coupled motion of the cold masses can therefore be neglected.

During machine operation, horizontal orbit vibration at frequencies around $10 \mathrm{~Hz}$ can be detected in both rings $[1,2]$. The amplitude of this beam jitter corresponds to 5 to 10 percent of the rms beam size for a $6 \sigma$ normalized

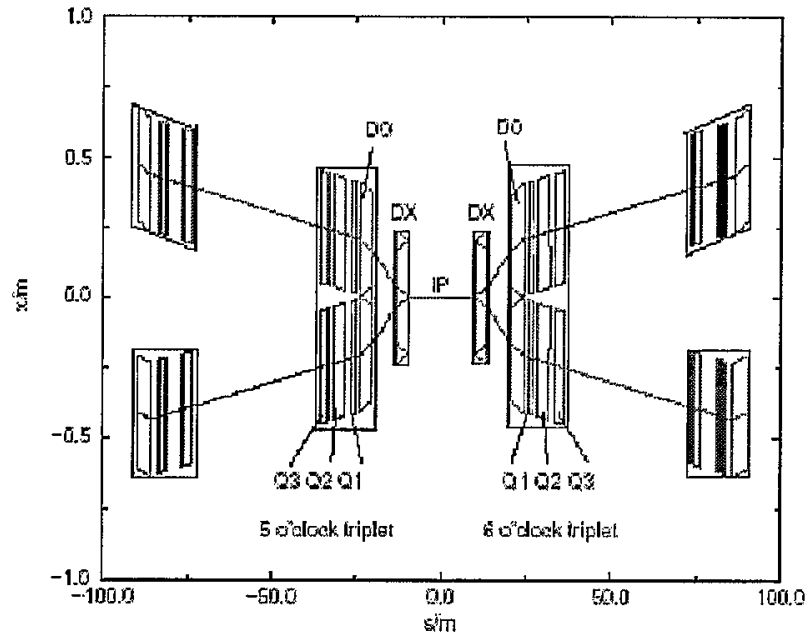

FIGURE 2. Schematic view of a RHIC interaction region lattice. emittance of $\varepsilon=10 \pi \mathrm{mm}$ mrad at any BPM around the ring, pointing at multiple noise sources. A spectral analysis of this orbit jitter also revealed that spectra in both beams are practically identical, indicating common vibration sources in both rings.

Since the two RHIC rings share only very few common magnetic elements while the D0 dipoles and the arcs are completely independent, it was soon found that the beam jitter was caused by mechanical vibration of the interaction region triplets. Taking into account the RHIC optics it was shown that the observed jitter amplitudes cannot be explained by the amplitudes measured on the triplet cryostats, but is rather caused by independent vibration of the various cold masses within the cryostat.

Since this beam jitter may potentially lead to emittance growth and therefore luminosity degradation due to modulated beam-beam offsets at the interaction points, it is very desirable to find and eliminate its source. We therefore calculated and measured mechanical vibration frequencies of a triplet. In a very simplified mechanical model, the mechanical resonance frequencies of the cold mass for Q1, Q2, and Q3 are 15.8, 14.3, and 14.1 $\mathrm{Hz}$ that are close to those observed on the beam. A direct measure of the mechanical resonance frequencies by attaching an accelerometer to the magnet and a mechanical excitation revealed the dominant frequency lines at 9.75, 14.0, 16.0, and $19.5 \mathrm{~Hz}$.

\section{TRIPLET COOLING}

To investigate the possible origin of the observed triplet vibrations, helium pressure oscillations in the RHIC cryogenic system were measured. The purpose of this investigation is to verify the triplet vibration if it is introduced by any helium flow oscillation in the cooling loops of RHIC cryogenic system, to catch the pressure

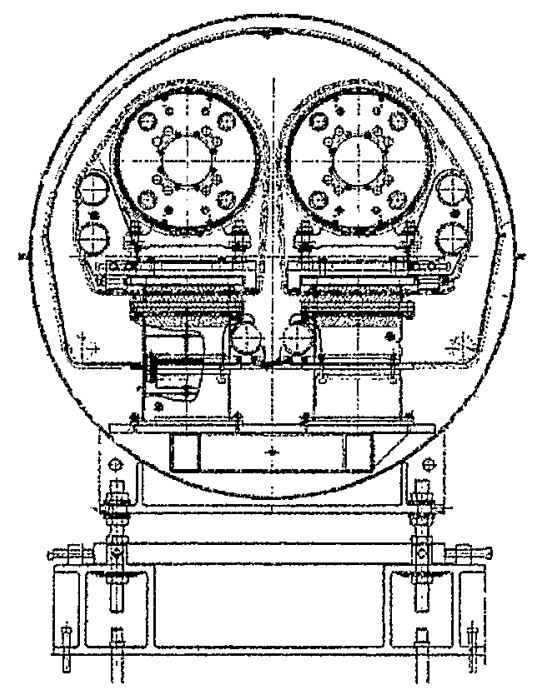

FIGURE 3. Cross section of RHIC triplet cryostat. oscillation in a range of $10 \mathrm{~Hz}$, if any, and to find 


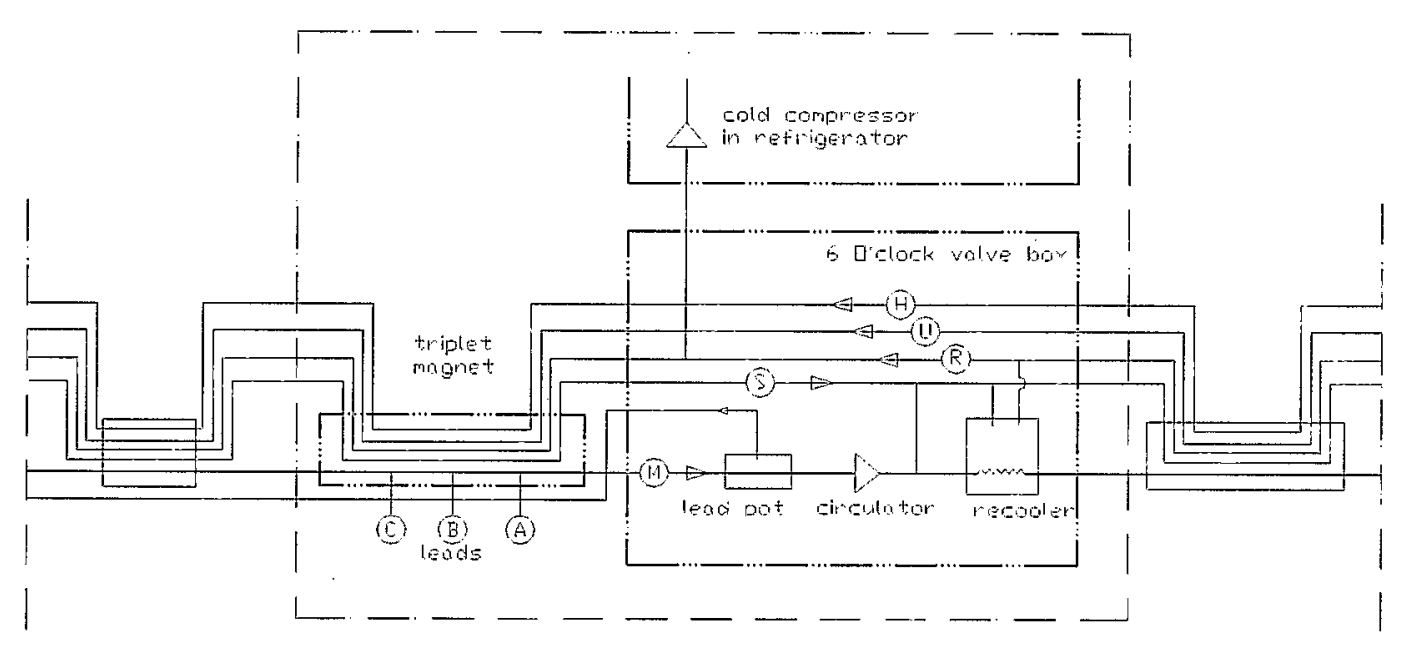

FIGURE 4. Flow diagram of RHIC triplet cryostat and valve box at 6 o'clock.

the cryogenic oscillation if it is related.

A schematic flow diagram of RHIC triplet cryostat and valve box is shown in FIGURE 4. The triplet cryostat contains the five helium transfer lines, supply (S), return $(\mathrm{R})$, utility $(\mathrm{U})$, thermal shield $(\mathrm{H})$, and magnet $(\mathrm{M})$ lines. The operating temperature in magnet line is $4.5 \mathrm{~K}$ and the pressure is 5 bar. The pressure drop in the magnet cooling loop is $0.25 \sim 0.5$ bar. The mass flow rate is $100 \mathrm{~g} / \mathrm{s} \sim 150 \mathrm{~g} / \mathrm{s}$. A mechanical circulating compressor in each ring is used to sustain the closed loop flow. These compressors are located at the 6 o'clock valve boxes. And a mechanical cold expander is installed in the refrigerator for pressure reduction in the recooler. Three power lead ports for each ring are extended out locally at the triplet cryostat. New pressure transducers were installed in the five helium transfer lines $(S, R, U, S, M)$ at the $6 o^{\prime}$ clock valve box and at the six power lead ports of the triplet cryostat.

\section{PRESSURE MEASUREMENTS}

The pressure measurements were carried out at different conditions of cryogenic operation, which involve cryogenic test procedures by changing the helium mass flow rates, the flow direction, and the loop pressures in certain cryogenic transfer lines. The running rpm of helium circulators was also adjusted along with the cryogenic valves at a scale within the safety margin of normal operation. These "cold-only" tests were performed when the triplet magnets were cold but not powered to prevent unnecessary magnet quenches. The "power-on" pressure measurements for each transfer line were conducted at valve box area when the magnets were energized as well as when beam was on.

The measured pressures for five transfer lines are grouped in FIGURES 5 to 6 in two different time scales, one-minute data group in FIGURE $\mathbf{5}$ and one-second data group in FIGURE 6. Two pressure oscillations were observed in the $U$ line and $M$ line, respectively. One in the $U$ line was at a low frequency of $0.1 \mathrm{~Hz}$ and had the peak-to-peak pressure (pkpk) of $0.25 \mathrm{kPa}$ at the base pressure of 0.68 bar. The one in the $\mathrm{M}$ line at the valve box was at a high frequency of $10.5 \sim 10.8 \mathrm{~Hz}$ and had the pkpk pressure of $6.5 \sim 6.8 \mathrm{kPa}$ at the base pressure of 4.9 bar. The one also in the lead ports extended from the magnet line at triplet cryostat was about $5 \sim 7 \mathrm{~Hz}$ at pkpk pressure of $2.7 \sim 5.5 \mathrm{kPa}$. At the lead ports some 
intensity changes in each ring but at the same frequency. Also some excited and decay and periodic pattern were observed in the lead ports, see FIGURES 7 and 8. There were no pressure oscillations found in the other three cryogenic lines. There were also no pressure oscillations found when the magnet was warm in both Blue and Yellow rings.
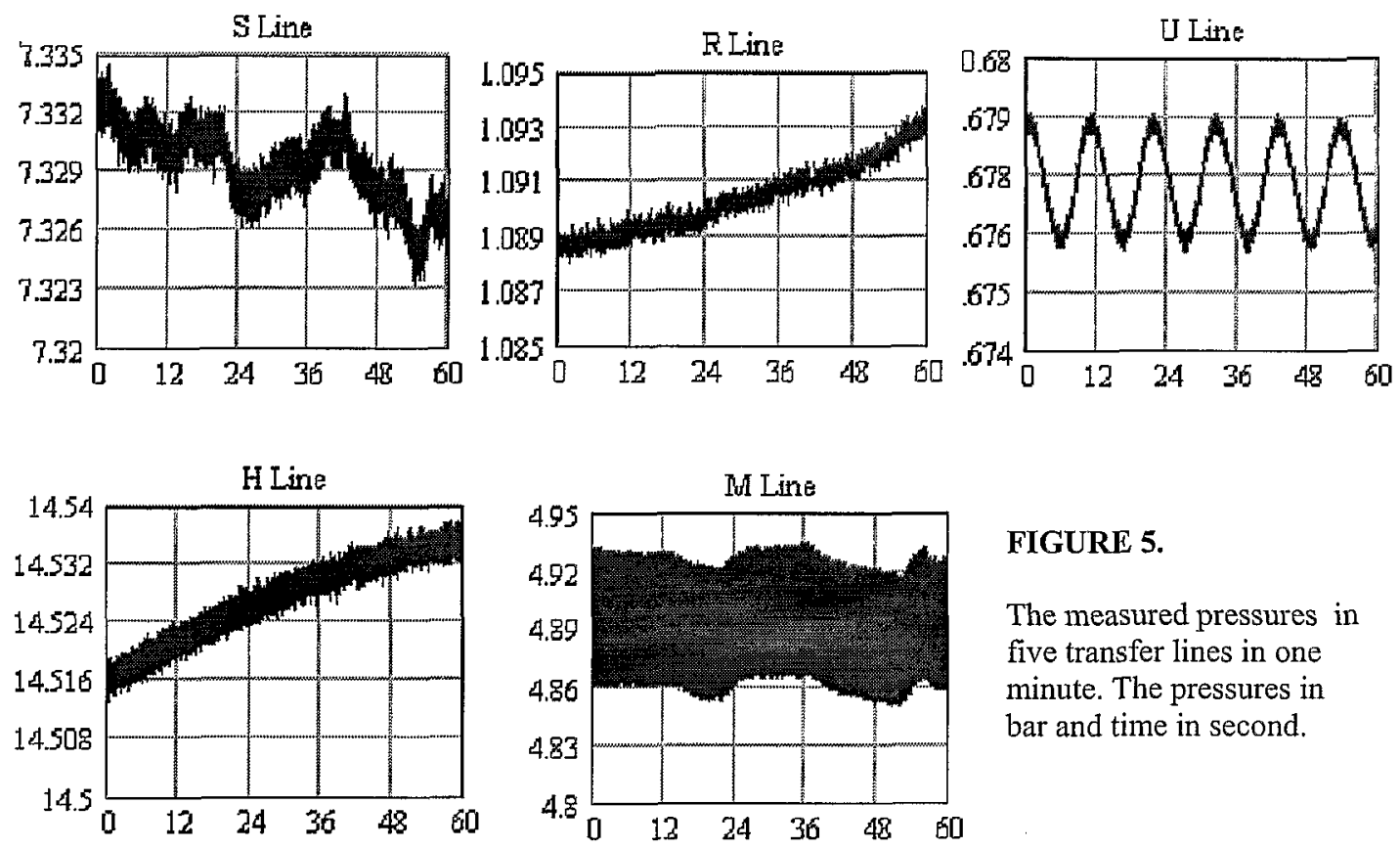

\section{FIGURE 5.}

The measured pressures in five transfer lines in one minute. The pressures in bar and time in second.
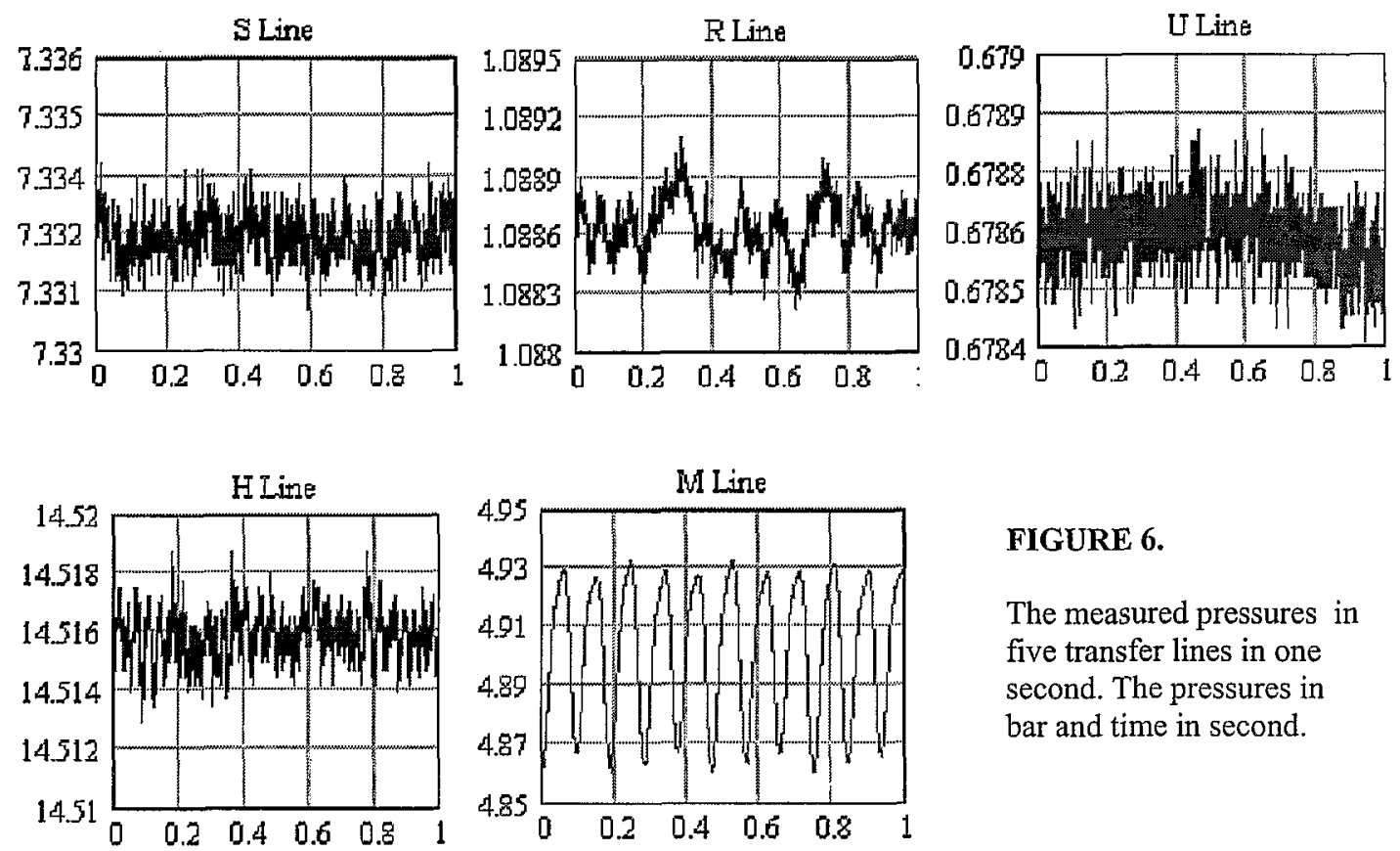

\section{FIGURE 6.}

The measured pressures in five transfer lines in one second. The pressures in bar and time in second. 


\section{DISCUSSIONS}

It is interesting to see that these pressure oscillation patterns in the $U$ line and $M$ line were not significantly changed with the base pressure and mass flow rate. It is also interesting to see the regular harmonic pattern of each oscillation that is so different from the typical pattern of "cryogenic oscillations" which were observed in many cryogenic systems. Those typical cryogenic oscillations include the thermal acoustic oscillations, the "Geyser" oscillations, gasification oscillations, and those oscillations under the periodic heat load. FIGURE 9 shows an example of cryogenic oscillation of helium pressure and temperature in a cryogenic system at BNL.

These observations led to the investigation of two mechanical devices in the system, the cold expender installed in the $U$ line for pressure reduction in the recooler and the cold compressor installed in the $\mathrm{M}$ line for helium flow circulation. It was observed, however, that no changes in its intensity and frequency when circulator changed speed $(3500 \sim 6000$ $\mathrm{rpm}$ ). There is also no direct correlation with flow conditions regulated by manipulating the associated cryogenic valves.
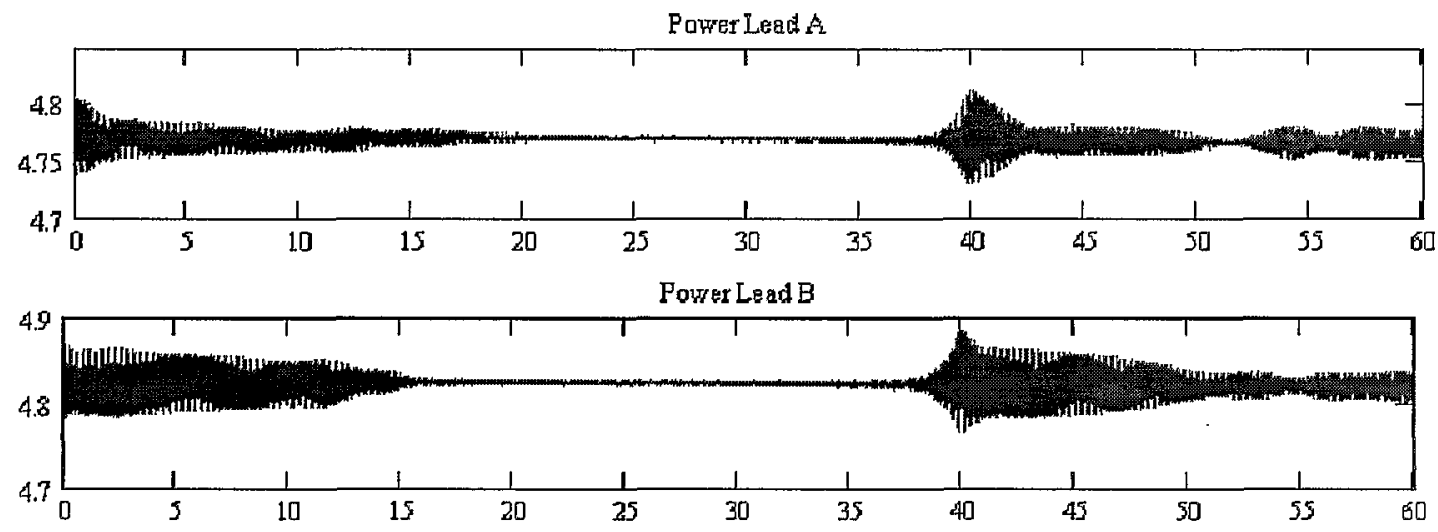

FIGURE 7. Pressure oscillations in the lead ports shown some excited and decay patterns.
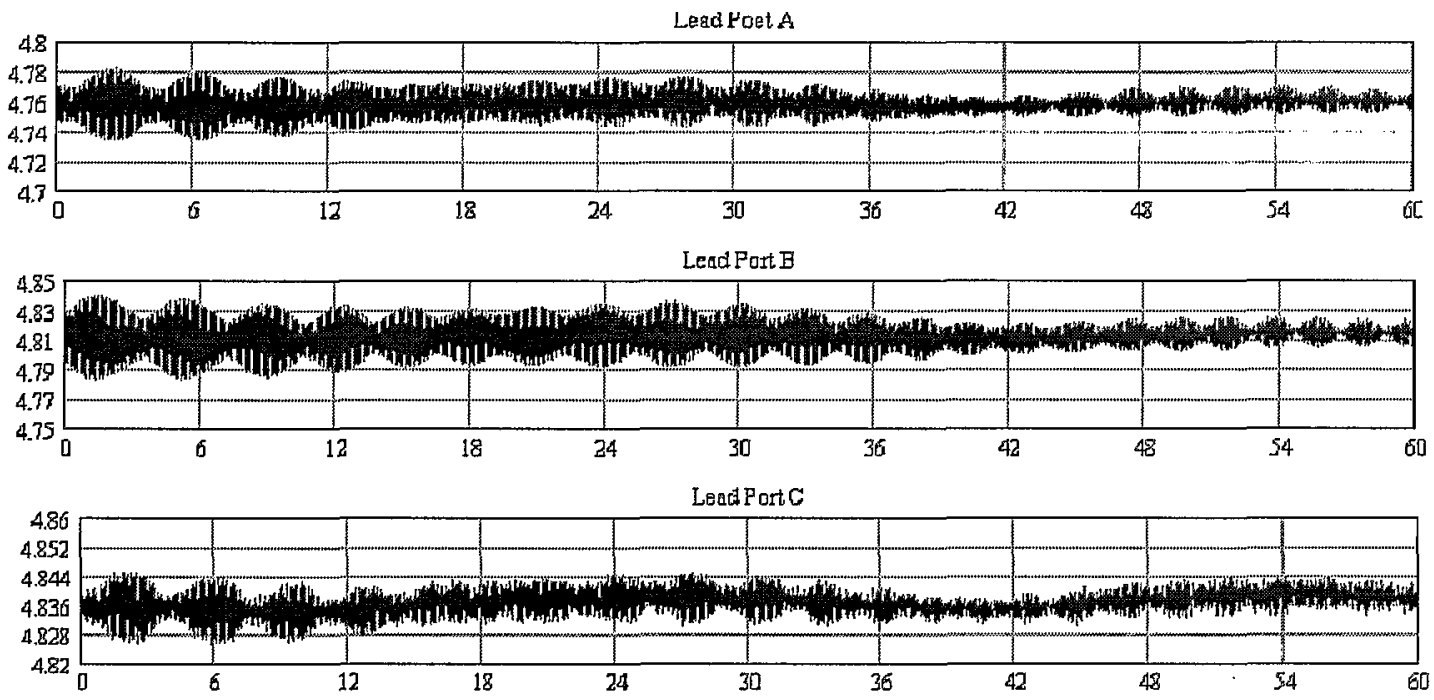

FIGURE 8. Pressure oscillations in the lead ports shown some periodic patterns. 

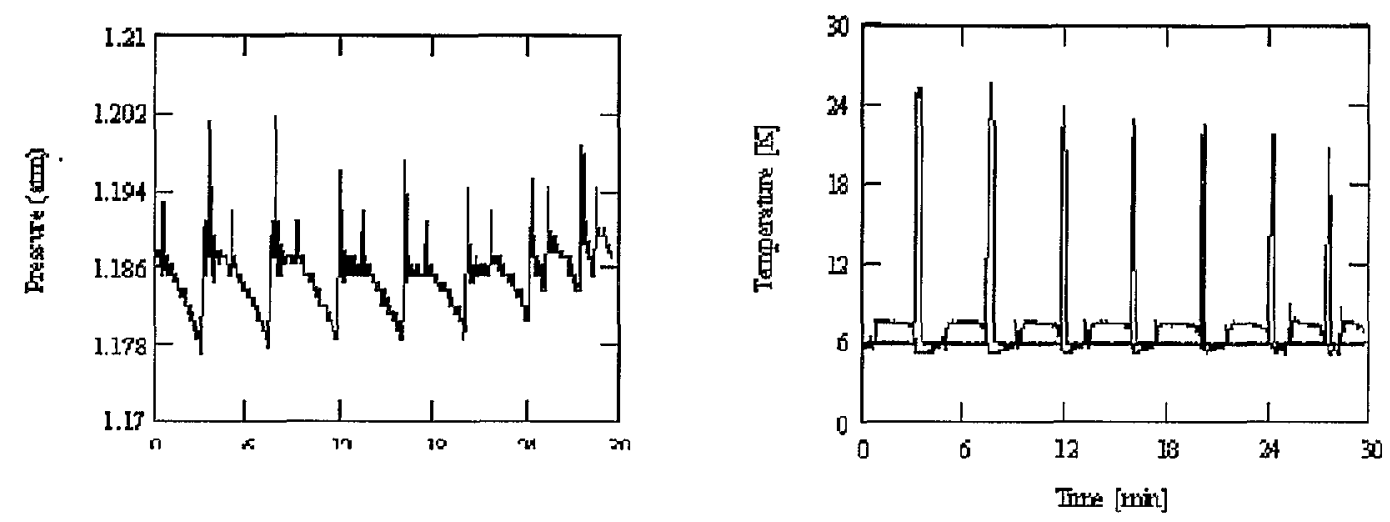

FIGURE 9. Pressure oscillations in the lead ports shown some excited and decay patterns.

\section{CONCLUSION}

Two pressure oscillations were observed in the helium transfer lines of the RHIC triplet magnets. Coincidently, both of these two cooling loops involve the mechanical rotating devices, the cold expander and the cold circulator. However, though the frequency of this oscillation is very close to the mechanical vibration detected on the triplet cryostat as well the orbit jitter frequency of the RHIC beam, it could not yet be positively confirmed as the root cause of the magnet vibration and the resulting orbit oscillations. Further investigation to find and eliminate the sources is underway.

\section{ACKNOWLEDGMENTS}

Work performed under Contract Number DE-AC02-98CH10886 with the U.S. Department of Energy.

\section{REFERENCES}

1. Montag, C., Brennan, M., Butler, J., Bonati, R., P. Koello, "Measurements of Mechanical Triplet Vibrations in RHIC," in Proc. EPAC2002, Paris

2. Montag, C., Ganetis, G., Jia, L., Louie, W., "Vibration Studies on a Superconducting RHIC Interaction Region Quadrupole Triplet" in Proc. PAC2003, to be published. 